\title{
Generation of recombinant Orf virus using an enhanced green fluorescent protein reporter gene as a selectable marker
}

Zhangyong Ning ${ }^{1,2+}$, Yongzheng Peng ${ }^{1,3+}$, Wenbo Hao ${ }^{4 \dagger}$, Chaohui Duan ${ }^{1,5}$, Daniel L Rock and Shuhong Luo ${ }^{1,4^{*}}$

\begin{abstract}
Background: Reporter genes are often used as a selectable marker for generation of recombinant viruses in order to investigate the mechanism of pathogenesis and to obtain candidate vaccine viruses. Routine selection of the recombinant parapoxvirus is time-consuming and labor intensive. Therefore, developing a novel method for selection is critical.

Results: In this study, we developed a rapid method to generate recombinant Orf viruses (ORFV) based on the enhanced green fluorescent protein (EGFP) reporter gene as a selectable marker. The coding sequence of EGFP gene was amplified from pEGFP-N1 vector and subcloned into the pZIPPY-neo/gus plasmid under the control of the early-late vaccinia virus (VACV) W7.5 promoter and flanked by two multiple cloning sites (MCS) to generate a novel transfer vector pSPV-EGFP. Using the pSPV-EGFP, two recombination cassettes pSPV-113LF-EGFP-113RF and pSPV-116LF-EGFP-116RF were constructed by cloning the flanking regions of the ORFV113 and ORFV116 and inserted into two MCS flanking the EGFP gene. Using this novel system, two single gene deletion mutants OV-

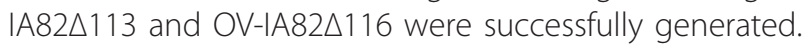

Conclusions: This approach shortens the time needed to generate recombinant ORFVs (rORFVs). Thus, the pSPVEGFP vector provides a direct, fast, and convenient way to manipulate the recombinant viruses, indicating that it is highly suited for its designed purpose.
\end{abstract}

\section{Background}

Orf virus (ORFV), the prototypic member of the Parapoxviridae, is the cause of a papular demertitis in sheep and goats known as contagious ecthyma [1]. ORFV has been used in veterinary medicine as a preventive and therapeutic immunomodulatory agent. Live or inactivated ORFV preparations exhibit dose-dependent immunomodulatory effects when administered to multiple animal species including cattle, horses, cats and dogs. Significant therapeutic efficacy of ORFV preparations in preventing or treating stress-associated and other infectious disease conditions has been well documented [1-4]. However, ORFV functions (genes/proteins/ mechanisms of action) associated with modulation and

\footnotetext{
* Correspondence: shluo815@yahoo.com

+ Contributed equally

'Department of Pathobiology, College of Veterinary Medicine, University of Illinois at Champaign-Urbana, 2001 S. Lincoln Avenue, Urbana, IL 61802, USA Full list of author information is available at the end of the article
}

manipulation of host immune responses are still poorly understood.

Previously we identified 16 novel, mostly terminally located ORFV genes with putative virulence and host range functions [5], hypothesizing that these proteins perform novel but undescribed immunomodulatory functions in the host. To investigate the role of these genes during virus infection, one approach to studying uncharacterized genes is to create mutations and/or deletions of specific ORFV genes to disrupt their function. This can be achieved by using transfer vectors designed to insert into site-specific locations of the viral genome via homologous recombination [6]. However, the experimental procedures to generate and isolate rORFVs are adapted from standard protocols used in generation of the vaccinia virus. This protocol is labor intensive and time consuming $[7,8]$.

The Green Fluorescent Protein (GFP) was discovered by Shimomura et al as a companion protein to aequorin

\section{Biomed Central}


[9], the famous chemiluminescent protein from Aequorea jellyfish which exhibits bright green fluorescence when exposed to blue light $[10,11]$. In cellular and molecular biology, the GFP gene is frequently used as a reporter of expression [12]. Lately, the Enhanced Green Fluorescent Protein (EGFP) was developed and has been introduced and expressed in many bacteria [13], yeast and other fungi [14], fish [15], plants [16], flies [17] and mammalian cells [18] including human. In recent years, recombinant GFP/EGFP expression coupled with flow cytometry to produce an individual cell-based readout with increased sensitivity has been widely utilized to generate recombinant vaccinia viruses to study virus tropism and a high-throughput vaccinia virus neutralization assay [19-22].

In the present study, to shorten the time required to generate rORFVs, we took advantage of EGFP reporter gene as a selection marker to isolate rORFVs. The novel transfer vector pSPV-EGFP was constructed to express EGFP in rORFVs. Using this novel system, two singlegene deletion mutants OV-IA82 $\Delta 113$ and OVIA $82 \Delta 116$ were successfully generated. This new approach allowed us to generate the desired rORFVs in less than 15 days. Compared to the neo/gusA selection, EGFP selection provides a direct, fast and convenient way to construct recombinant viruses, indicating the usefulness of the pSPV-EGFP vector.

\section{Results}

Generation of rORFVs using neo/gusA selection

To generate rORFVs, the convenient and efficient methods for identifying recombinants are necessary. In recent years, to improve the efficiency of recombinant isolation, the combination of drug selection and identifiable color detection provides an advantage over previous methods in the poxvirus field [7]. To investigate the functions of these novel genes encoded by ORFV, construction of gene-deleted mutant viruses is a first step. In previous studies [23-25], the pZIPPY-neo/gus vector containing the neo/gusA cassette was initially used to construct recombinant cassettes based on the flanking regions of ORFV002, ORFV012, ORFV024, ORFV113, ORFV116, ORFV120 and ORFV121. The preliminary results were summarized in Table 1 . Using this strategy, four single gene deleted-mutant recombinants OV-IA82 $\Delta 002$ [23], OV-IA82 $\Delta 024$ [24], OV-IA82 $\Delta 120$ (unpublished data), OV-IA $82 \Delta 121$ [25] were finally generated and isolated through 17 and 20 rounds of plaque purification (each round takes at least 4 or 5 days to pick blue plaques, Table 1). However, OV-IA $82 \Delta 012$, OV-IA $82 \Delta 113$, and OV-IA $82 \Delta 116$ are still contaminated with the parent virus after more then 20 rounds of plaque purification (Table 1). This protocol is labor intensive and time consuming, requiring at least 3 or 4 months to isolate and
Table 1 Comparison of generating rORFVs using the pSPV-EGFP and PZIPPY-neo/gus vectors

\begin{tabular}{ccccc}
\hline $\begin{array}{c}\text { OV } \\
\text { gene }\end{array}$ & \multicolumn{2}{c}{ Rounds of plaque assay } & \multicolumn{2}{c}{$\begin{array}{c}\text { Time to obtain the puried } \\
\text { rOVs (days) }\end{array}$} \\
\cline { 2 - 5 } & $\begin{array}{c}\text { pSPV- } \\
\text { EGFP }\end{array}$ & $\begin{array}{c}\text { pZIPPY-neo/ } \\
\text { gus }\end{array}$ & $\begin{array}{c}\text { pSPV- } \\
\text { EGFP }\end{array}$ & $\begin{array}{c}\text { pZIPPY-neo/ } \\
\text { gus }\end{array}$ \\
\hline ORFV002 & $-^{a}$ & 16 & - & $80[23]^{\mathrm{b}}$ \\
ORFV012 & - & 20 & - & $>100^{\mathrm{C}}$ \\
ORFV024 & - & 19 & - & $95[24]$ \\
ORFV113 & 4 & 20 & 12 & $>100^{\mathrm{C}}$ \\
ORFV116 & 5 & 20 & 14 & $>100^{\mathrm{C}}$ \\
ORFV120 & - & 17 & - & 85 \\
ORFV121 & - & 18 & - & $90[25]$
\end{tabular}

${ }^{a}$ Not tested; ${ }^{b}$ Reference; ${ }^{c}$ After 20 rounds of plaque assay, still contaminated with wild type virus.

purify recombinants. A new strategy to generate rORFVs is critical.

\section{Construction of recombinant cassettes with the novel transfer vector $\mathrm{pSPV}$-EGFP}

To shorten the time needed to generate rORFVs, the novel transfer vector pSPV-EGFP (Figure 1A) was constructed using the EGFP reporter gene to replace the neo/gusA cassette [7]. To compare the efficiency of plaque purification between pZIPPY-neo/gusA [7] and pSPV-EGFP, two ORFV proteins, ORFV113 and ORFV116, were used for this study. Two transfer vectors, pSPV-113LF-EGFP-113RF and pSPV-116LF-EGFP116RF, were constructed using pSPV-EGFP. These cassettes encode EGFP under the control of the early-late VV 7.5 promoter, which allows for isolation and purification of recombinant viruses by using fluorescent signals under a fluorescent microscope. This novel experimental strategy streamlines the procedure. The experimental steps in this new strategy include: (i) generating the OV-IA $82 \Delta 113$ and OV-IA $82 \Delta 116$ after transfection of the pSPV-113LF-EGFP-113RF and pSPV116LF-EGFP-116RF vectors into ovine fetal turbinate (OFTu) cells previously inoculated with OV-IA82; (ii) visual monitoring of infected cultures by fluorescent microscopy to assess the level of rORFV infection; (iii) determine the optimal time (usually 24 hours during the second round of limited dilution) to harvest cells for plaque assay (Figure 1B).

\section{Generation of OV-IA82 $\Delta 113$ and OV-IA82 $\Delta 116$ using EGFP selection}

When the pSPV-113LF-EGFP-113RF and pSPV-116LFEGFP-116RF vectors were transfected into OFTu cells that had been previously exposed to OV-IA82 with 1.0 multiplicity of infection (MOI), spots of brightly EGFP-positive cells appeared within $6 \mathrm{~h}$, and strong fluorescent signal was observed under fluorescent 


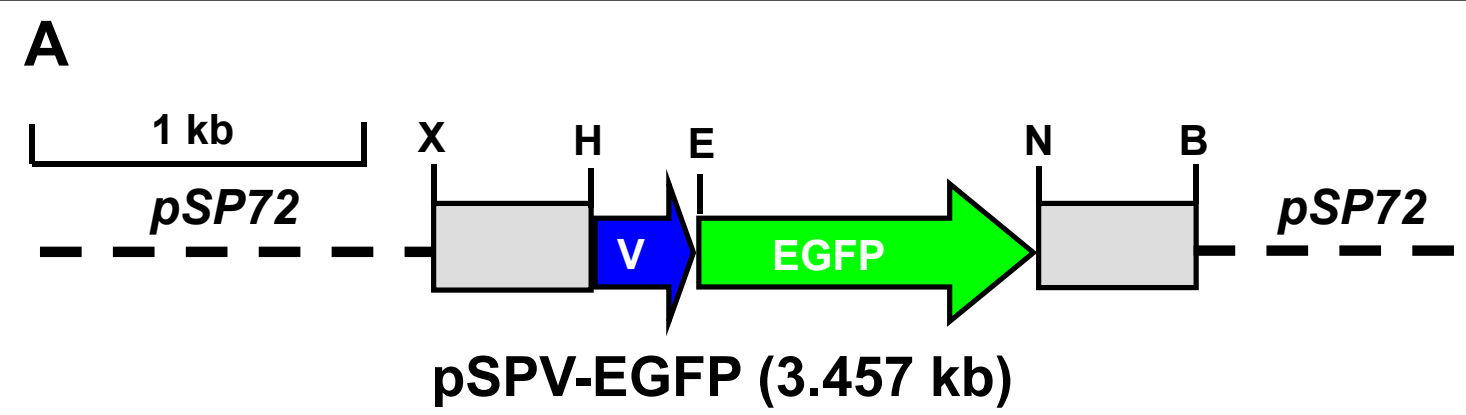

B

\section{(1) Infection}
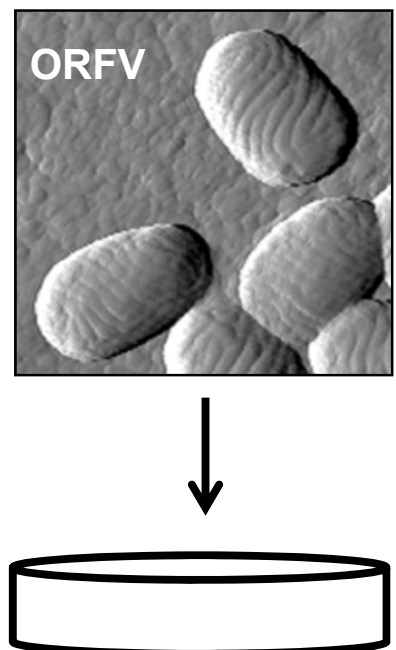

(2) Transfection

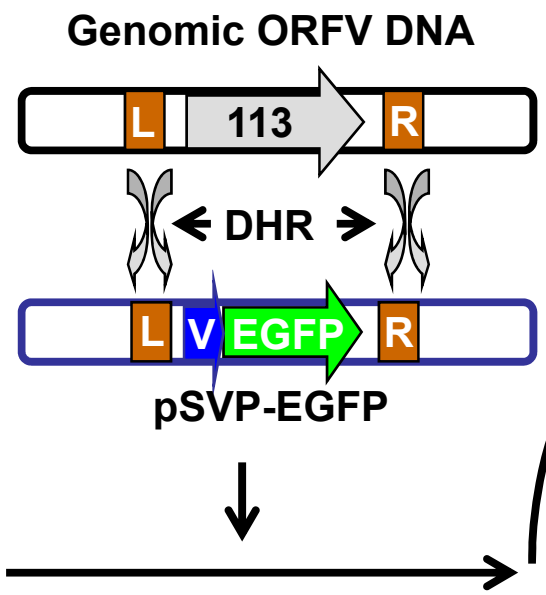

(3) Isolation

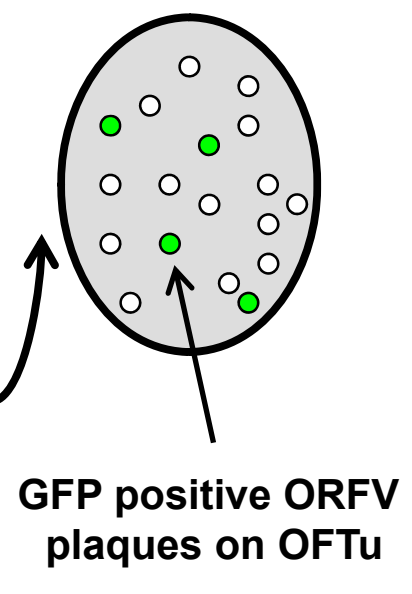

Figure 1 Infection/transfection scheme for generation of recombinant ORFV. A. Construction of the recombinant transfer vector pSPVEGFP. A cassette of selectable markers of E.coli neo and gusA genes in PZIPPY-neo/gus vector was replace by the EGFP reporter gene amplified from pEGFP-N1 vector (Clontech, CA) to generate the recombinant vector pSPV-EGFP. B. Generation of recombinant ORFV. OFTU Cells are infected with OV-IA82 and transfected with the transfer vector PSPV-113LF-EGFP-113RF. The resultant virus mixture is then plated on OFTu cells to eliminate OV-IA82 and the desired viruses were isolated. MCS: Multiple cloning sites. B: Bglll; E: EcoRl; H: Hindlll; N: Notl; and X: Xhol. V: vaccinia virus (strain WR) W early/late protomer Wp7.5. L: Up stream of ORFV113 un-transcription region; R: Down stream of ORFV113 untranscription region. DHR: double homologous recombination.

microscope after $24 \mathrm{~h}$. Cytopathic effect (CPE) of OFTU cells infected with OV-IA82 113 or OVIA $82 \Delta 116$ (Figure $2 \mathrm{~A}$ ) was observed after $24 \mathrm{~h}$ during the second round of limited dilution, and abundant EGFP expression was observed in infected OFTu cells. EGFP-positive cells in 96-well plates with dilutions higher than 1:10, 000 were harvested for further plaque purification. After two rounds of limited dilutions, three different individual recombinant clones for each protein were selected to run 2 or 3 times plaque assays to remove parent virus contamination. Plaques with a fluorescent signal can be identified in OFTu cells after $12 \mathrm{~h} \mathrm{pi}$, and individual green plaques were picked under fluorescent microscope at $36 \mathrm{~h}$ pi (Figure 2B). This demonstrates that EGFP selection substantially shortens the time than conventional protocols such as neo/gusA selection.

\section{Identification of OV-IA82 $\Delta 113$ and OV-IA82 $\Delta 116$}

To determine the purity of OV-IA $82 \Delta 113$ and OVIA $82 \Delta 116$, PCR was conducted using primers designed from internal coding regions of ORFV113 and ORFV116 or EGFP (Table 2). The results demonstrated that the EGFP gene was amplified from three different clones of OV-IA $82 \Delta 113$ (Figure $3 \mathrm{~A}$ ) and OV-IA82 116 (Figure $3 B)$, but not from OV-IA82 or mock infected cells. In 
A

GFP
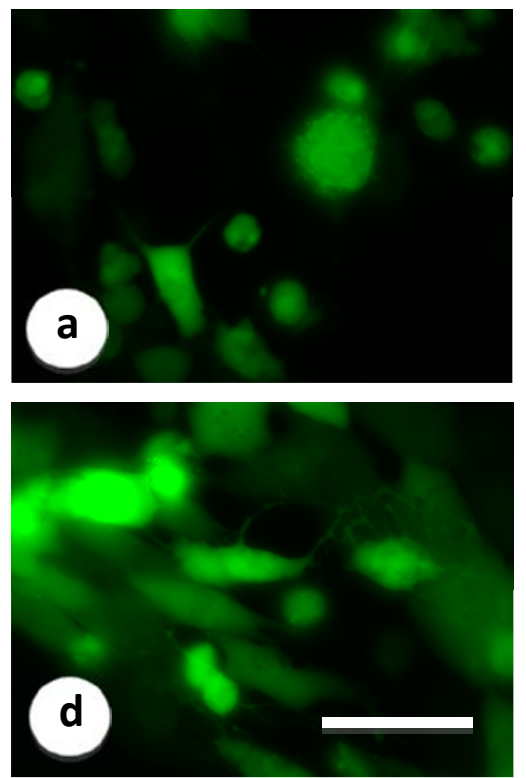

b
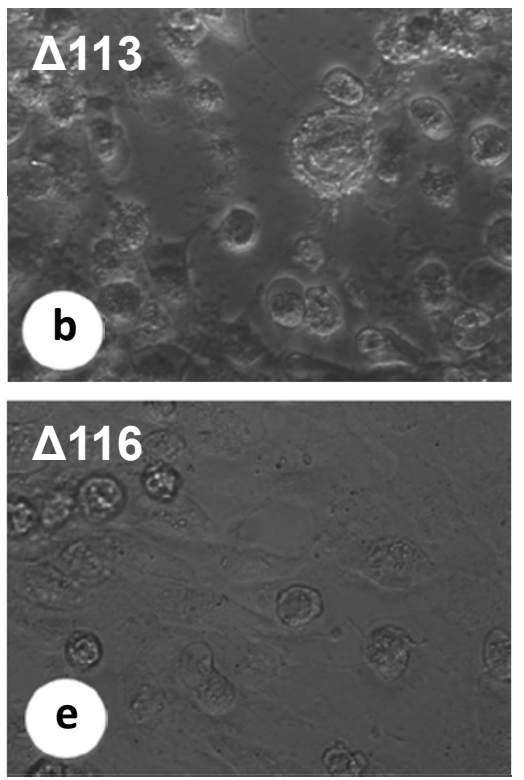

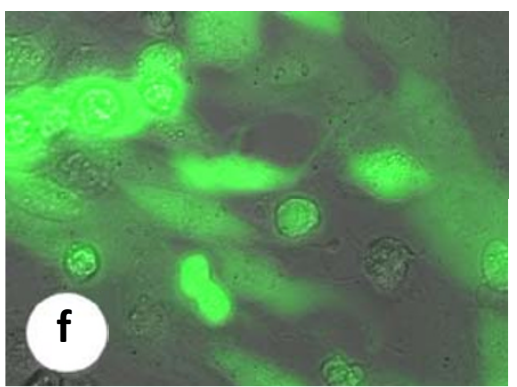

Overlay

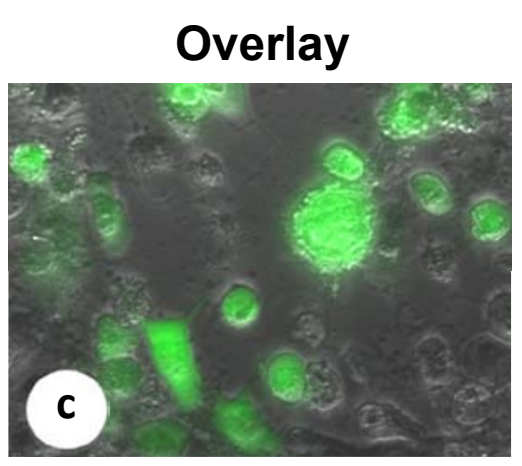

B

GFP
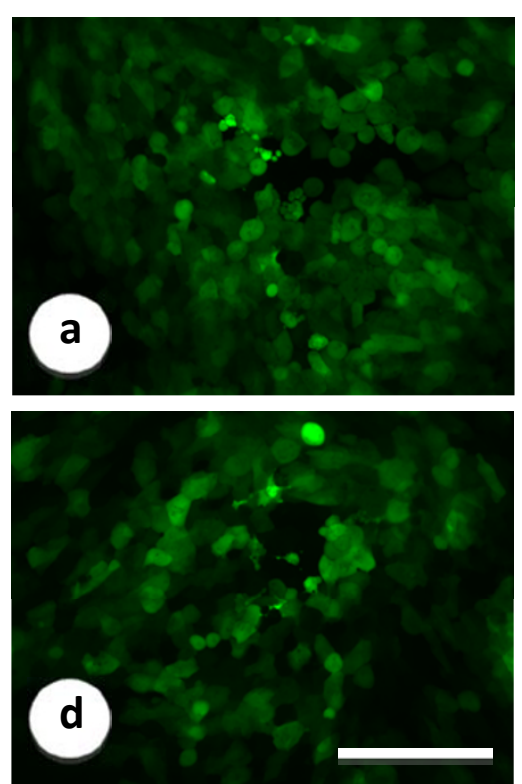

BF
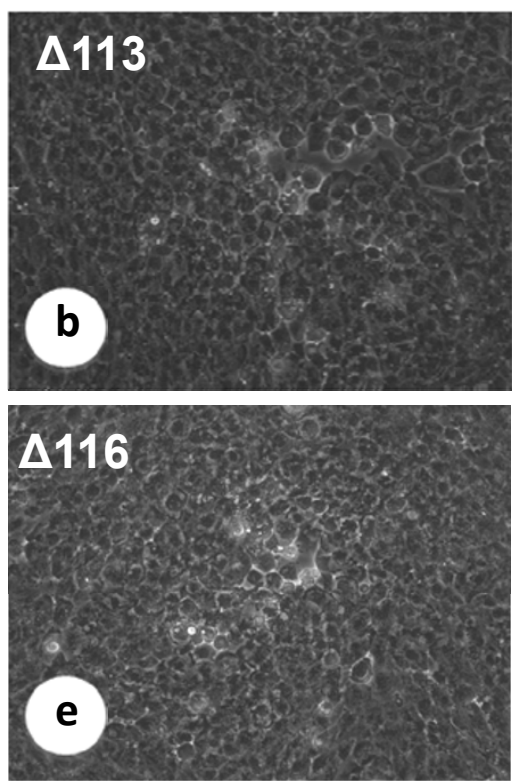

Figure 2 Fluorescent microscopy showing the cytopathic effects (CPE) of OFTu cells infected with OV-IA82 113 and OV-IA82 1116 by

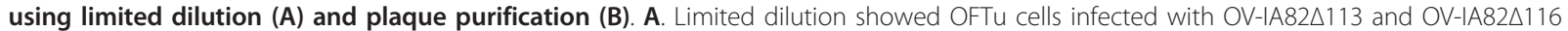
after $24 \mathrm{~h}$ pi. The strong fluorescent signal was observed in OFTu cells infected with both OV-IA82 113 and OV-IA82 116 . (b) and (e) show the same fields as in (a) and (d) by bright-field microscopy. (c) and (f), overlay of (a) and (b) or (d) and (e). Cells were visualized in a Leica SP2 microscope in $63 \times$ magnification. Bar, $10 \mu \mathrm{m}$. B. Plaque purification showed the plaques with strong fluorescent signal in OFTu cells infected with OV-IA82 $113(\mathrm{a}, \mathrm{b}, \mathrm{c})$ and OV-IA82 $116(\mathrm{~d}, \mathrm{e}, \mathrm{f})$ at $36 \mathrm{~h}$ pi during the second round of plaque purification. (b) and (e) show the same fields as in (a) and (d) by bright-field microscopy. (c) and ( $\mathrm{f}$ ), overlay of (a) and (b) or (d) and (e). Cells were visualized in a Leica DMI4000B inverted microscope in 10x magnification. Bar, $20 \mu \mathrm{m}$. 
Table 2 Oligonucleotide primers and restriction enzymes

\begin{tabular}{|c|c|c|}
\hline Primer & Sequence & Restriction enzyme* \\
\hline GFPFW1 & 5'-AACTTAGAATTCGCCACCATGGTGAGCAAGGGCGA-3' & EcoR I \\
\hline GFPRv1 & 5'-ATCAATGCGGCCGCTTACTTGTACAGCTCGTCCA-3' & Not I \\
\hline GFPintrFw2 & 5'-GACGTAAACGGCCACAAGTT-3' & \\
\hline GFPintrRv2 & 5'-ACTGGGTGCTCAGGTAGTGG-3' & \\
\hline GFPseqFw3 & 5'-CGACCACTACCAGCAGAACA-3' & \\
\hline GFPseqRv3 & 5'-AAGTCGTGCTGCTTCATGTG-3' & \\
\hline 113LFFw1 & 5'-AGGCCTCTAAGCTTCAGGTTCCGGCTTCAGATGCGCGT-3' & Hind III \\
\hline 113LFRv1 & 5'-ATTCGCGTCGACCACCAACACTTCCATTGTTGCGGC-3' & Sal I \\
\hline 113RFFw2 & 5'-TCTTATGCGGCCGCGAGCCGCCGATGCAGATCGAGGTA-3' & Not I \\
\hline 113RFRv2 & 5'-ATTCGCAGATCTTCGATCGCCAGTGCGCGGCGCATG-3' & $\mathrm{Bgl} \|$ \\
\hline 113intrFw3 & 5'-CGCCGTAATATGCTTAACCGGAGC-3' & \\
\hline 113intrRv3 & 5'-CGGACCGTGTTGGTCGTTGGGTCT-3' & \\
\hline 113seqFw4 & 5'-TTAGCTTCCTTGTTITTATC-3' & \\
\hline 113seqRv4 & 5'-GTCCTTCGGGTCAGAGTCC-3' & \\
\hline 116LFFw1 & 5'-GCCTCTACTAGTAGGAAGTGGCCTCGCCGACCACGA-3' & Spe I \\
\hline 116LFRv1 & 5'-ATTCGCGTCGACGTGGATGTCTCTAAGGTTCAATAC-3' & Sal I \\
\hline 116RFFw2 & 5'-TCTTATGCGGCCGCCTACCACTGGTACCAGCACCTCCT-3' & Not I \\
\hline 116RFRv2 & 5'-ATTCGCAGATCTGGCGCTACAGGCGTCCTGCAGGAA-3' & $B g l \|$ \\
\hline 116intrFw3 & 5'-GAACAACACGTCAACCGATG-3' & \\
\hline 116intrRv3 & 5'-AGGTGTGGGTTGACTTCCAG-3' & \\
\hline 116 seqFw4 & 5'-GTCGAGCAGATGTTCATGGA-3' & \\
\hline 116seqRv4 & 5'-ATGCTGCACTTCCTGGAGAT-3' & \\
\hline 001 intrFw1 & 5'-CTCGGTGACCTGCCTGAC-3' & \\
\hline 001 intrRv1 & 5'-CTCGCGCACGTCGTAGAT-3' & \\
\hline
\end{tabular}

* Restriction enzyme sites are underlined in prime sequences

contrast, the ORFV113 and ORFV116 genes were amplified from OV-IA82 infected cells only (Figure 3).

To verify ORFV113 and ORFV116 genes were successfully deleted from OV-IA82 via the transfer vector pSPV-EGFP and the EGFP gene was incorporated into the OV-IA82 genome by homologous recombination;
Southern blot analysis was performed on genomic DNA isolated from OV-IA82, OV-IA $82 \triangle 113$ and OVIA $82 \Delta 116$. The ORFV113 probe detected the ORFV113 gene in OV-IA82 (Figure 4A lower panel, lane 1) but not in OV-IA82 113 (Figure 4A lower panel, lane 2). The ORFVO01 gene is the only double copy located at

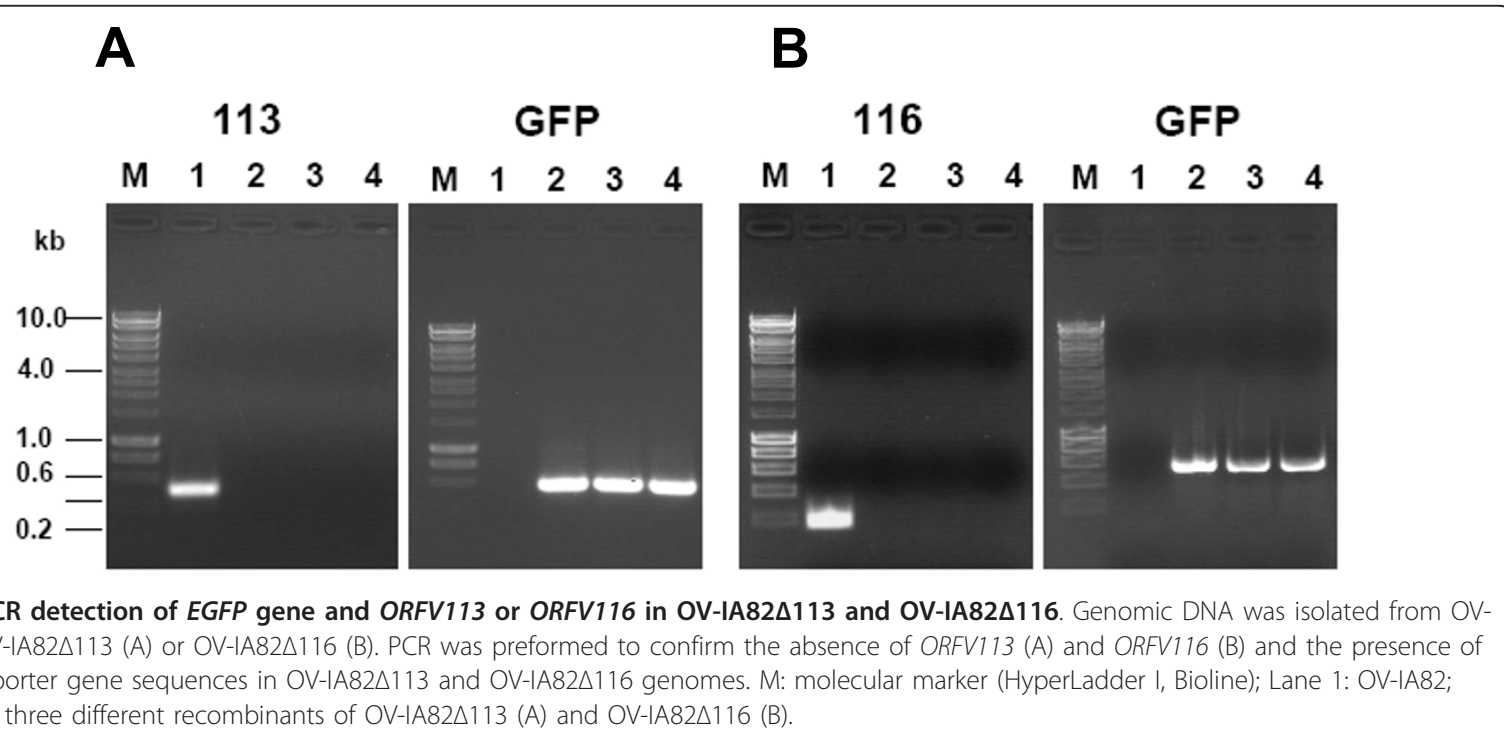




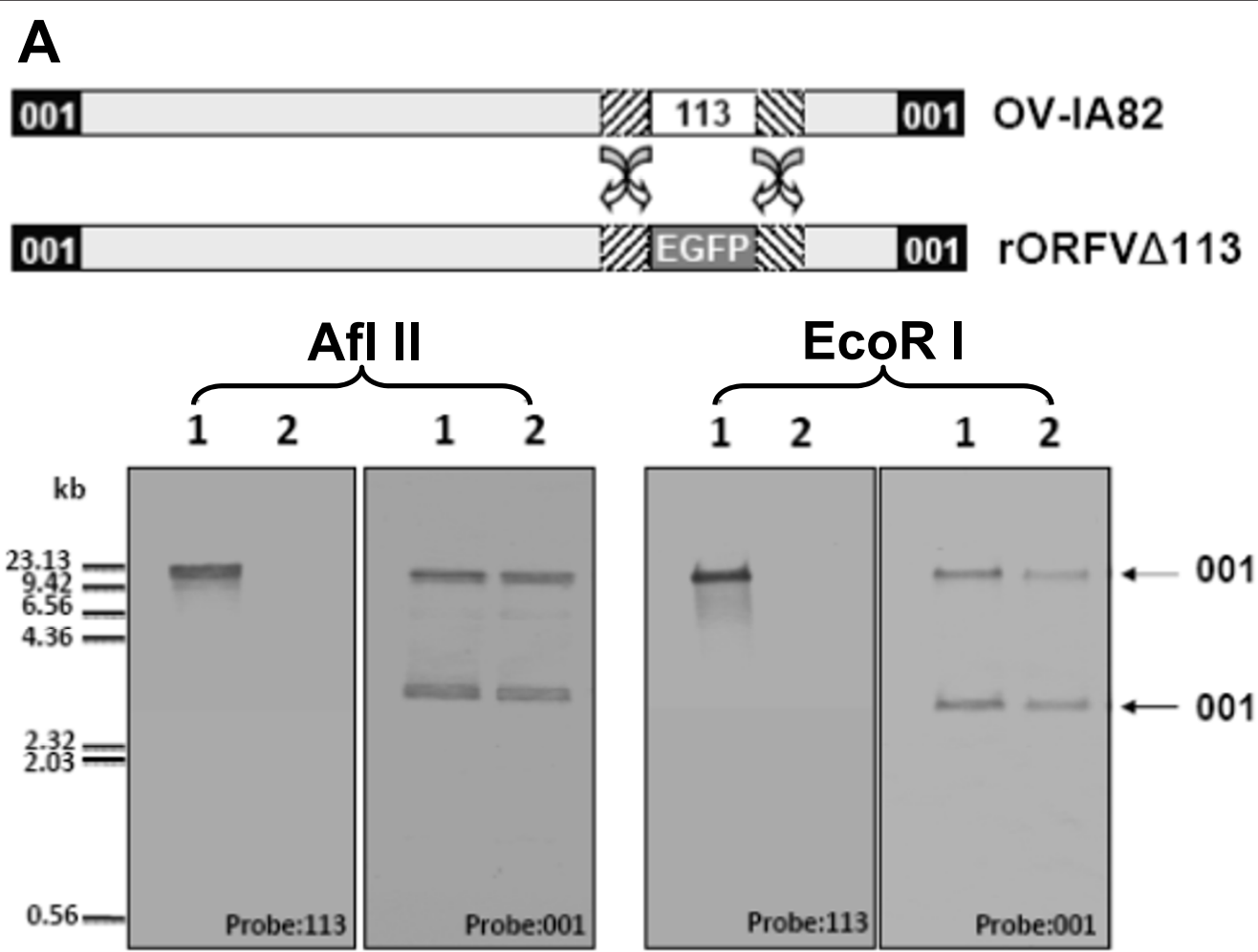

B

001001001 OV-IA82

AfI II

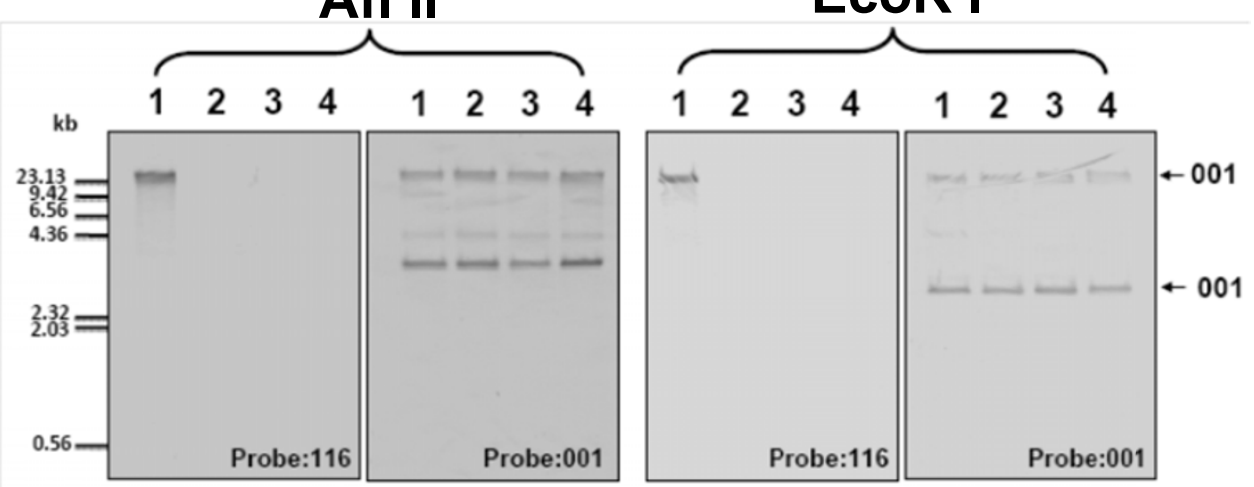

Figure 4 Characterization of mutants of OV-IA82 2113 and OV-IA82 2116 by Southern blotting. A. OV-IA82 113 . Upper panel is a schematic of OV-IA82 genome before and after removal of the 113 gene using the deletion vector, pSPV-EGFP by double homologous recombination to generate gene-deletion mutant OV-IA82 113 . Lower panel shows Southern blot analysis. Genomic DNA was isolated from OVIA82 (lane 1) and OV-IA82 113 (lane 2) and digested with restriction enzyme AfllI or EcoRI respectively. The 113 internal probe was unable to detect the 113 gene in the recombinant OV-IA82 113 genome, indicating that the 113 gene was completely removed from the 113 locus of the genome. The 001 probe detected both end of the 001 loci in both OV-IA82 and OV-IA82 113 genomes. B. OV-IA82 116 . Upper panel A is a schematic of OV-IA82 genome before and after removal of the 116 gene using the deletion vector, pSPV-EGFP by double homologous recombination to generate gene-deletion mutant OV-IA82A116. Lower panel shows the 116 gene, which was completely removed from the 116 locus of the OV-IA82 genome by Southern blot analysis. The 116 internal probe was unable to detect the 116 gene in the OV-IA82 116 genome. The 001 probe detected both end of sequences in OV-IA82 (lane: 1) and three different clones of OV-IA82 116 (lanes: 2 to 4). 
the left and right ends of the viral genome. The ORFV001 probe detected the same pattern in both OVIA 82 and OV-IA $82 \Delta 113$, indicating that the deletion of ORFV113 does not affect the left and right ends of the viral genome. The EGFP probe detected the EGFP gene in OV-IA82 $\Delta 113$ but not in OV-IA82 (data not shown). Similar results were obtained when the ORFV116 probe was applied (Figure 4B). Sequencing of left and right flanking regions of the deleted gene, which were involved in the recombination, confirmed the integrity of parental virus sequences in the mutant virus (data not shown).

\section{Deletion of ORFV113 and ORFV116 do not affect ORFV replication in vitro}

To investigate the infectivity of the gene-deleted mutant viruses, a one-step growth curve was conducted. Replication properties of OV-IA $82 \Delta 113$, OV-IA $82 \Delta 116$, and OV-IA82 were compared after infection of OFTu cells. No significant differences in growth were detected between the mutant and wild type viruses $(\mathrm{P}>0.05)$ in OFTu cells, indicating that ORFV113 and ORFV116 are not essential for ORFV replication in OFTu cells (Figure 5). The data also demonstrates that expression of EGFP in OV-IA $82 \Delta 113$ and OV-IA $82 \Delta 116$ does not affect ORFV growth and replication in vitro. Also shown in our previous report [23], the exogenous expression of EGFP did not affect the viral phenotype and the biological function in ORFV002 revertant virus (OVIA 82 Rv002GFP) which was constructed by using the pSPV-EGFP system. Taken together, the pSPV-EGFP vector could be widely used for recombination and foreign gene expression studies.

\section{Discussion}

We have shown that generating rORFVs is easy and efficient using the EGFP reporter gene and is faster than conventional neo/gusA methods. Using this novel system, two single gene deletion mutants OVIA $82 \Delta 113$ and OV-IA $82 \Delta 116$ were successfully generated. Additional gene deletion mutants such as ORFV001, ORFV005, ORFV012 and ORFV124 are under way. The advantages of EGFP fluorescent selection compared to conventional strategies are as follows: (i) easy to construct recombinant cassettes using the pSPV-EGFP; (ii) visual monitoring of infected cells by fluorescent microscopy to assess the level of rORFV infection; (iii) easy to determine the optimal time to harvest cells; (iv) streamlining the procedures and shortening the time needed to isolate and purify the plaques (less than 15 days), compared to 3 or 4 months using neo/gusA method (Table 1); (v) picking plaques directly under fluorescent microscope or by fluorescence activated cell sorting (FACS) within $24 \mathrm{~h}$ pi, no antibiotics and substrates needed; (vi) using the pSPV-EGFP plus the pZIPPY-neo/gus allows to make a double gene deletion mutants for multiple copy genes or the same cluster of gene family. Moreover, EGFP expression in infected cells provides a reliable, straightforward methodology to maintain viral stocks free from contamination with spontaneous ORFV revertants by the rapid selection marker of GFP-positive cells. Furthermore, the growth curve demonstrate that EGFP expression in recombinant viruses does not affect the virus replication (Figure 5) and the luciferase assay results of our previous reported OVIA82Rv002GFP [23] also showed that the EGFP expression does not affect the virus biological functions. Previous studies have shown that recombinant vaccinia virus-mediated EGFP expression has been used to monitor infection in vitro [19] or in vivo [26]. Our EGFP-based vector construct may represent a practical tool to study ORFV infection and pathogenesis in natural hosts.

\section{Conclusions}

In this study, the pSPV-EGFP vector, which contains two MCSs for insertion ORFV genes or foreign genes, provides a reliable, straightforward methodology for the advanced studies of ORFV replication, host range, as well as foreign gene expression. Using this new approach to generate and isolate rORFVs based on the EGFP selection marker remarkably reduces the time for recombinant virus isolation and purification. In addition, EGFP localization will give the opportunity to study host-virus interaction at the cellular level in vivo. Taken together, the novel transfer vector pSPV-EGFP provides an efficient and convenient way for gene deletion from OV-IA82 genome to study the novel gene functions and may also be used to acquire gene-deleted mutants from other viruses.

\section{Methods}

\section{Cells and viruses}

Primary ovine fetal turbinate (OFTu) cells were kindly provided by Dr. Howard D. Lehmkuhl (USDA) and were maintained in minimal essential medium (MEM) supplemented with $10 \%$ fetal bovine serum (FBS), 100 $\mu \mathrm{g} / \mathrm{ml}$ streptomycin, $100 \mathrm{U} / \mathrm{ml}$ penicillin, $50 \mu \mathrm{g} / \mathrm{ml}$ gentamicin and $2 \mathrm{mM}$ L-glutamine.

ORFV IA82 (OV-IA82) strain was isolated from lamb nasal secretion during an orf outbreak at the Iowa Ram Test station in 1982, and has been fully sequenced [5]. Low passage OV-IA82, which is fully virulent, was used to construct deletion mutants OV-IA82 1113 (Figure $4 \mathrm{~A}$ ) and OV-IA-82 $\Delta 116$ (Figure $4 \mathrm{~B}$ ) and was used here in all procedures involving infections with wild type virus, PCR amplification and cloning of viral genes. The 


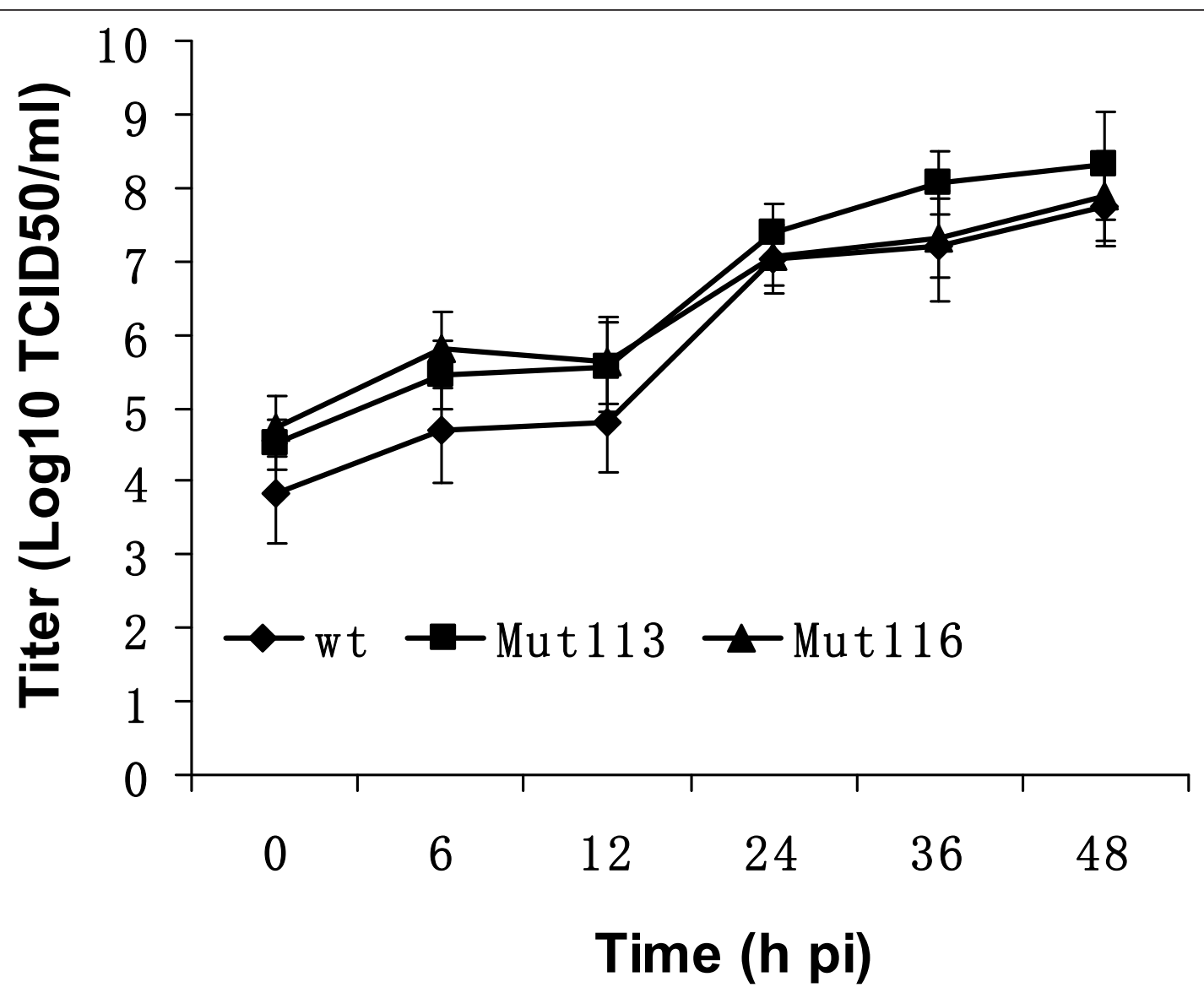

Figure 5 Replication characteristics of OV-IA82 1113 and OV-IA82 116 in OFTu cells. One step growth curves were performed in OFTu cells. Statistics showed that there was no significant difference between wild type and mutant viruses $(P>0.05)$. Data represent the averages of the results of three independent experiments. Error bars show standard deviations. Diamond: OV-IA82; Square: OV-IA82 $\triangle 113$; Triangle: OV-

IA82 $\triangle 116$.

virus was propagated in OFTu cells and was purified by sucrose gradient ultra-centrifugation before storage at $-80^{\circ} \mathrm{C}$ for use.

\section{Construction of pSPV-EGFP}

To obtain the EGFP gene sequence, two primers were designed, sense-primer GFPFw1 and antisense-primer GFPRv1. An EcoR I site was added to the sense-primer and Not I to the antisense-primer (Table 2). Thermostable high fidelity DNA polymerase and dNTPs were obtained from Invitrogen (Carlsbad, CA, USA). Polymerase chain reaction (PCR) was performed in $50 \mu \mathrm{l}$ thin-wall polypropylene tubes (Denville Scientific, NJ, USA) using a PTC-200 DNA Engine thermal cycler (Bio-Rad, Shelton, CA, USA) under the following conditions: denaturation at $96^{\circ} \mathrm{C}$ for $2 \mathrm{~min}$ followed by 30 cycles of denaturation at $94^{\circ} \mathrm{C}$ for $45 \mathrm{~s}$, annealing at $54^{\circ}$ $\mathrm{C}$ for $45 \mathrm{~s}$, elongation at $72^{\circ} \mathrm{C}$ for $1 \mathrm{~min}$, and a final extension at $72^{\circ} \mathrm{C}$ for $7 \mathrm{~min}$. PCR products were visualized in $1 \%$ agarose gel with ethidium bromide staining, cut out, and purified using a Qiagen gel extraction kit (Qiagen, Valencia, CA, USA). The products were then cloned into vector pZIPPY-neo/gus [7], which had been linearized with EcoRI and NotI (New England Biolabs, Ipswich, MA, USA) to generate the novel transfer vector pSPV-EGFP (Figure 1A). The plasmid was propagated in Escherichia coli strain Top10 (Invitrogen, Carlsbad, CA, USA). DNA sequencing of the pSPV-EGFP was done to ensure that the correct construct had been obtained. The complete DNA sequence of the pSPVEGFP was deposited in GenBank under the accession number: GU062789.

\section{Construction of recombinant cassettes}

DNA preparations and manipulations were performed using standard methods as described by Sambrook et al. [27] or followed instructions from manufactures. Two recombination cassettes were constructed by PCR amplifying ORFV113 and ORFV116 left and right flanking regions from OV-IA82 genome using primers listed 
in Table 2. The PCR products were cloned into the pSPV-EGFP MCSs to generate pSPV-113LF-EGFP113RF and pSPV-116LF-EGFP-116RF. In addition, to compare the efficiency between the pSVP-EGFP and pZIPPY-neo/gus, seven recombinant cassettes were also constructed using pZIPPY-neo/gus vector. The ORFV002, ORFV012, ORFV024, ORFV113, ORFV116, ORFV120 and ORFV121 left and right flanking regions from OV-IA82 genome were amplified and cloned into the pZIPPY-neo/gus MCSs, flanking the neomycin resistance (neo) and $\beta$-glucuronidase reporter (gus) genes under control of the VACV VV7.5 and modified H5 promoters, respectively [7]. The resulting recombinant vectors were named pZNG-ORFV002 [23], pZNGORFV012, pZNG-ORFV024 [24], pZNG-ORFV113, pZNG-ORFV116, pZNG-ORFV120, pZNG-ORFV121 [25]. All sub-cloning procedures were confirmed by using restriction enzymes and DNA sequence analysis.

\section{Generation of recombinant viruses}

OV-IA $82 \Delta 113$ and OV-IA $82 \Delta 116$ viruses were constructed by infecting OFTu cells (in T25 flasks) at a multiplicity of infection (MOI) of 1.0 with wild type OV-IA 82 for 3 hours and subsequently transfecting the cells with $10 \mu \mathrm{g}$ of pSVP-113LF-EGFP-113RF and pSVP-116LF-EGFP-116RF transfer vectors by standard in vivo recombination protocols $[28,29]$. Transfections were carried out using Lipofectamine 2000 (Invitrogen, Carlsbad, CA, USA) according to the manufacturer's instructions.

Viruses were harvested $48 \mathrm{~h}$ pi by scraping infected/ transfected OFTu cells into sterile $15 \mathrm{ml}$ conical tubes. The cell suspensions were vortexed, frozen/thawed 3 times, and then centrifuged at $1000 \mathrm{rpm}$, for $10 \mathrm{~min}$ at $4{ }^{\circ} \mathrm{C}$ (Eppendorf Centrifuge 5810R, 15 amp version, Hamburg, Germany). The supernatant (viruses) were transferred into $2 \mathrm{ml}$ cryogenic vials (Corning, NY, USA) and stored at $-80^{\circ} \mathrm{C}$ for future use.

In order to select and purify recombinant viruses, limited dilution and plaque assays were performed. $3 \times 10^{4}$ of OFTu cells per well were seeded into 96-well plates one day before infection. On the second day, the cells were infected with serial 10-fold dilutions of viruses from $10^{-1}$ to $10^{-11}$ (900 $\mu \mathrm{l} 1 \times \mathrm{MEM}+100 \mu \mathrm{l}$ of virus). The cells were examined under a fluorescent microscope (Leica DMI4000B inverted microscope) $12 \mathrm{~h}$ after inoculation. The cells exhibiting fluorescent signal with dilutions higher than 1:10, 000 were collected for the second round of limited dilution. Typically, after $24 \mathrm{~h}$ pi, strong green fluorescent signal was observed in 96well plates. The cell suspensions were harvested and frozen/thawed as above. The supernatant (viruses) was stored at $-80^{\circ} \mathrm{C}$ for use.
After 2 or 3 rounds of limited dilution, plaque assays were carried out for further purification. $7 \times 10^{5}$ of OFTu cells per well were passed into 6-well plates one day before infection. On the next day, cells were infected with serial dilutions of viruses from $10^{-1}$ to $10^{-}$ 6 . The viruses were allowed to absorb to the cells for 1 $\mathrm{h}$ at $37^{\circ} \mathrm{C}$, in $5 \% \mathrm{CO}_{2}$ incubator. The medium was removed and then the cells were overlaid with $3 \mathrm{ml}$ of MEM containing $5 \%$ of FBS and $0.5 \%$ low melting point agarose (Sea Kem ${ }^{\circledR}$ GTG $^{\circledR}$, Lonza, Rockland, ME, USA). Plaques with a GFP signal were visualized and picked 24 or $36 \mathrm{~h}$ pi. A minimum of 2 or 3 plaques were picked for further plaque purification. The last plaque purified recombinant viruses were expanded in $35 \times 10 \mathrm{~mm}$ dishes; genomic DNA was extracted using QIAamp DNA blood kit (QIAGEN, Germany). PCR was performed to screen for the wild type virus contamination using internal primers from ORFV113 and ORFV116 coding regions (Table 2). PCR conditions were the same as described above except that the annealing temperature was based on the Tm values of each primer. Southern blotting was further carried out to confirm that double homologous recombination had occurred.

For comparison studies, OV-IA $\Delta 002$, ORFV $\Delta 012$, OVIA-82 $\Delta 024, \quad$ OV-IA $82 \Delta 113, \quad$ OV-IA $82 \Delta 116, \quad$ OV IA $82 \Delta 120$ and OV-IA $82 \Delta 121$ were also constructed using neo/gus selection following the procedures as described above. The only difference is that those recombinant viruses produced blue plaques (gusA activity) in the presence of X-gluc (Clontech, Palo Alto, CA, USA). Blue plaques were picked at day 4 or day 5 pi.

\section{Southern blots}

Genomic DNA was extracted from OV-IA82, OVIA $82 \Delta 113$ and OV-IA $82 \Delta 116$ viruses, respectively. One microgram of viral DNA was digested with AflII or EcoRI (New England Biolabs, Inc.), electrophoresed via a $1.0 \%$ agarose gel and transferred via capillary action to a nylon membrane (Bio-Rad, CA) using standard protocols [30]. The membranes were hybridized with specific digoxin (Roche, Mannheim, Germany) labeled probes, which were generated specifically for the ORFV113, ORFV116, ORFV001 and EGFP genes. The probes for ORFV113, ORFV116, EGFP and ORFV001 were amplified by using internal primers (Table 2), same primers applied for PCR detection as described above. Amplicons were purified and labeled with digoxin followed the manufacture's instruction (Roche, Mannheim, Germany). To determine the ends of recombinant viruses, the same blots were stripped with N, N-dimethylformamide (Sigma, St. Louis, MO, USA) at $50-60^{\circ} \mathrm{C}$ for $1 \mathrm{~h}$, until color had been removed completely. During the stripping process, the solution needed to be changed 
every $20 \mathrm{~min}$. The stripped membrane was incubated in stripping buffer $(0.2 \mathrm{M} \mathrm{NaOH}$ and $0.1 \%$ sodium dodecyl sulfate) for $30 \mathrm{~min}$ at $37^{\circ} \mathrm{C}$, and re-hybridized with a specific digoxin labeled probe that were generated toward the ORFV001 gene cassette. The NBT/BCIP Detection kit (Roche, Mannheim, Germany) for nonradioactive color development was used for hybridization analysis of Southern blots according to the manufacture's instructions.

\section{Sequencing analysis}

To determine homologous recombination, three plaques, which were confirmed by PCR detection using ORFV113 and ORFV116 internal primers (113intrFw3 and 113intrRv3 or 116intrFw3 and 116intrRv3, table 2) were applied for sequencing analysis. Two pairs of primers (Table 2), 113seqFw4 and GFPseqRv3; GFPseqFw3 and 113seqRv4 or 116seqFw4 and GFPseqRv3; GFPFw3 and $116 s e q R 4$, were utilized to amplify the regions involved in recombination. PCR conditions were the same as described above except that the annealing temperature was employed based on the Tm values of each primer. The PCR products were cloned into pCR2.1 TA cloning plasmid (Invitrogen, CA) for sequencing analysis using an Applied Biosystems PRISM 3730 automated DNA sequencer (Applied Biosystems, Foster City, CA, USA).

\section{Virus expansion, titration, and growth curve}

Based on the Southern blot and sequence analysis, one clone of OV-IA $82 \Delta 113$ and OV-IA $82 \Delta 116$ were used for expansion. Confluent monolayers of OFTu cells in four T150 $\mathrm{cm}^{2}$ flasks were infected at MOI of 1 . The viruses were harvested when all cells were rounded but still attached (about 3 or 4 days pi). The cell suspensions were prepared as above. The supernatants were dispensed into $2 \mathrm{ml}$ cryogenic vials $(1 \mathrm{ml} / \mathrm{vial})$ and stored at $-80^{\circ} \mathrm{C}$ for future use.

96-well plates of $90 \%$ confluent monolayers OFTu cells were prepared as above. Rows of cells were infected with serial 10-fold dilutions of wild type and mutant viruses of $10^{-1}$ to $10^{-9}$, with one row for control (no virus). One column was used for one dilution. CPE was read at $2 \mathrm{~d} \mathrm{pi}$, and the final read was carried out at $7 \mathrm{~d}$ pi. The median tissue culture infected dose $\left(\mathrm{TCID}_{50}\right)$ per $\mathrm{ml}$ was calculated using a spreadsheet.

One step growth curves were conducted as our pervious reports [23-25]. OFTu cells were prepared in $35 \times$ $10 \mathrm{~mm}$ dishes $\left(7 \times 10^{5}\right.$ cells/dish $)$ one day before the experiment. The cells were counted on the second day and were infected at a MOI of 10 with wild type and mutant virus. Virus was harvested at $0,6,12,24,36,48$ $\mathrm{h}$ pi and titrated. The growth curves were plotted as titer $\left(\log _{10} \mathrm{TCID}_{50} / \mathrm{ml}\right)$ versus time course $(\mathrm{h})$ pi. Data are presented as the mean \pm standard deviation from three independent experiments. $P$ values were determined by using the unpaired two-tailed Student's t-test. Statistical significance was set at the $95 \%$ confidence level.

\section{Acknowledgements}

We thank Diego G Diel (USDA, ARS, Southeast Poultry Research Laboratory) and Gustavo Delhon (Department of Veterinary and Biomedical Sciences) for the technique support. This work was supported by the grant (No. 31070138 and No. 31170147 to Shuhong Luo) from the National Natural Science Foundation of China (NSFC).

\section{Author details}

${ }^{1}$ Department of Pathobiology, College of Veterinary Medicine, University of Illinois at Champaign-Urbana, 2001 S. Lincoln Avenue, Urbana, IL 61802, USA. ${ }^{2}$ College of Veterinary Medicine, South China Agricultural University, Guangzhou 510642, People's Republic of China. ${ }^{3}$ Department of Laboratory Medicine, Zhujiang Hospital, Southern Medical University, Guangzhou, 510280, People's Republic of China. ${ }^{4}$ Institute of Antibody Engineering, School of Biotechnology, Southern Medical University, 1838 N. Guangzhou Avenue,Guangzhou, 510515, People's Republic of China. ${ }^{5}$ Laboratory of Clinical Immunology, the Sun Yat-Sen Memorial hospital, Sun Yat-Sen University, Guangzhou 510120, Guangdong, People's Republic of China.

\section{Authors' contributions}

$\mathrm{SL}, \mathrm{ZN}$ and DR participated in design of the study. $\mathrm{CD}$ and $\mathrm{WH}$ constructed the plasmid PSPV-EGFP. ZN, YP and WH prepared the recombinant virus and get the grow curve and Southern blot analysis. ZN, SL and DR analyzed the data and wrote the manuscript. All authors read and approved the final manuscript.

Received: 4 October 2011 Accepted: 22 December 2011

Published: 22 December 2011

\section{References}

1. Haig DM, Fleming S: Immunomodulation by virulence proteins of the parapoxvirus orf virus. Vet Immunol Immunopathol 1999, 72:81-86.

2. Rziha HJ, Henkel M, Cottone R, Meyer M, Dehio C, Büttner M: Parapoxviruses: potential alternative vectors for directing the immune response in permissive and non-permissive hosts. J Biotechnol 1999, 73:235-242.

3. Friebe A, Friederichs S, Scholz K, Janssen U, Scholz C, Schlapp T, Mercer A Siegling A, Volk HD, Weber O: Characterization of immunostimulatory components of orf virus (parapoxvirus ovis). J Gen Virol 2011, 92:1571-1584.

4. Sturgill TL, Giguère S, Franklin RP, Cohen ND, Hagen J, Kalyuzhny AE: Effects of inactivated parapoxvirus ovis on the cumulative incidence of pneumonia and cytokine secretion in foals on a farm with endemic infections caused by Rhodococcus equi. Vet Immunol Immunopathol 2011 140:237-243.

5. Delhon $\mathrm{G}$, Tulman ER, Afonso $\mathrm{CL}$, Lu Z, de la Concha-Bermejillo $\mathrm{A}$ Lehmkuhl HD, Piccone ME, Kutish GF, Rock DL: Genomes of the parapoxviruses ORF virus and bovine papular stomatitis virus. $J$ Virol 2004, 78:168-177.

6. Mackett M, Smith $G L$, Moss B: Vaccinia virus: a selectable eukaryotic cloning and expression vector. Proc Natl Acad Sci USA 1982, 79:7415-7419.

7. Dvoracek B, Shors T: Construction of a novel set of transfer vectors to study vaccinia virus replication and foreign gene expression. Plasmid 2003, 49:9-17

8. Lorenzo MM, Galindo I, Blasco R: Construction and isolation of recombinant vaccinia virus using genetic markers. Methods Mol Biol 2004, 269:15-30.

9. Shimomura O: Discovery of green fluorescent protein. Methods Biochem Anal 2006, 47:1-13.

10. Prendergast FG, Mann KG: Chemical and physical properties of aequorin and the green fluorescent protein isolated from Aequorea forskålea. Biochemistry 1978, 17(17):3448-3453.

11. Tsien RY: The green fluorescent protein. Annu Rev Biochem 1998, 67:509-544. 
12. Phillips GJ: Green fluorescent protein-a bright idea for the study of bacterial protein localization. FEMS Microbiol Lett 2001, 204:9-18.

13. Englert $\mathrm{DL}$, Jayaraman $\mathrm{A}$, Manson MD: Microfluidic techniques for the analysis of bacterial chemotaxis. Methods Mol Biol 2009, 571:1-23.

14. Kremer L, Baulard A, Estaquier J, Poulain-Godefroy O, Locht C: Green fluorescent protein as a new expression marker in mycobacteria. $\mathrm{Mol}$ Microbiol 1995, 17:913-922.

15. Peters KG, Rao PS, Bell BS, Kindman LA: Green fluorescent fusion proteins: powerful tools for monitoring protein expression in live zebrafish embryos. Dev Biol 1995, 171:252-257.

16. $\mathrm{Hu}$ W, Cheng CL: Expression of Aequorea green fluorescent protein in plant cells. FEBS Lett 1995, 369(2-3):331-334

17. Yeh E, Gustafson K, Boulianne GL: Green fluorescent protein as a vital marker and reporter of gene expression in Drosophila. Proc Natl Acad Sci USA 1995, 92:7036-7040.

18. Kishimoto H, Zhao M, Hayashi K, Urata Y, Tanaka N, Fujiwara T, Penman S, Hoffman RM: In vivo internal tumor illumination by telomerasedependent adenoviral GFP for precise surgical navigation. Proc Natl Acad Sci USA 2009, 106:14514-14517.

19. Domínguez J, Lorenzo MM, Blasco R: Green fluorescent protein expressed by a recombinant vaccinia virus permits early detection of infected cells by flow cytometry. J Immunol Methods 1998, 220:115-121.

20. Earl PL, Americo JL, Moss B: Development and use of a vaccinia virus neutralization assay based on flow cytometric detection of green fluorescent protein. J Virol 2003, 77:10684-10688.

21. Johnson MC, Damon IK, Karem KL: A rapid, high-throughput vaccinia virus neutralization assay for testing smallpox vaccine efficacy based on detection of green fluorescent protein. J Virol Methods 2008, 150:14-20.

22. Popov S, Mirshahidi S, Essono S, Song R, Wang X, Ruprecht RM: Generation of recombinant vaccinia viruses via green fluorescent protein selection. DNA Cell Biol 2009, 28:103-108.

23. Diel DG, Luo S, Delhon G, Peng Y, Flores EF, Rock DL: A nuclear inhibitor of NF-kappaB encoded by a poxvirus. J Virol 2011, 85(1):264-275.

24. Diel DG, Delhon G, Luo S, Flores EF, Rock DL: A novel inhibitor of the NF\{kappa\}B signaling pathway encoded by the parapoxvirus orf virus. $J$ Virol 2010, 84(8):3962-3973.

25. Diel DG, Luo S, Delhon G, Peng Y, Flores EF, Rock DL: Orf virus ORFV121 encodes a novel inhibitor of NF-kappaB that contributes to virus virulence. J Virol 2011, 85(5):2037-2049.

26. Norbury CC, Malide D, Gibbs JS, Bennink JR, Yewdell JW: Visualizing priming of virus-specific $\mathrm{CD}^{+}{ }^{+} \mathrm{T}$ cells by infected dendritic cells in vivo. Nat Immunol 2002, 3:265-271.

27. Sambrook J, Fritsch EF, Maniatis T: Molecular Cloning: a Laboratory manual Cold Spring harbour, Cold Spring harbour Press; 1989.

28. Earl PL, Moss B, Wyatt LS, Carroll MW: Generation of recombinant vaccinia viruses Curr Protoc Mol Biol; 2001

29. Shors T, Keck JG, Moss B: Down regulation of gene expression by the vaccinia virus D10 protein. J Virol 1999, 73:791-796.

30. Williams DL: The use of a PVDF membrane in the rapid immobilization of genomic DNA for dot-blot hybridization analysis. Biotechniques 1990, 8:14-15.

doi:10.1186/1746-6148-7-80

Cite this article as: Ning et al:: Generation of recombinant Orf virus using an enhanced green fluorescent protein reporter gene as a selectable marker. BMC Veterinary Research 2011 7:80.

\section{Submit your next manuscript to BioMed Central and take full advantage of:}

- Convenient online submission

- Thorough peer review

- No space constraints or color figure charges

- Immediate publication on acceptance

- Inclusion in PubMed, CAS, Scopus and Google Scholar

- Research which is freely available for redistribution

Submit your manuscript at www.biomedcentral.com/submit
Biomed Central 